\title{
Effects of the shape and Coriolis interaction in nuclear electromagnetic properties
}

\author{
Nikolay Minkov ${ }^{1, *}$ \\ ${ }^{1}$ Institute of Nuclear Research and Nuclear Energy, Bulgarian Academy of Sciences, Tzarigrad Road 72, BG-1784 Sofia, Bulgaria
}

\begin{abstract}
We study the manifestation of collective vibrations and rotations coupled to single-particle motion in odd-mass nuclei with the presence of axial quadrupole-octupole deformations. Our model incorporates $K$ mixing effects stemming from the Coriolis interaction between the odd nucleon and the even-even core and thus takes into account the probabilities for otherwise forbidden due to the axial symmetry electromagnetic transitions between excited states built on different intrinsic configurations. We demonstrate these effects in the quasi-parity-doublet spectrum of the nucleus ${ }^{221} \mathrm{Fr}$ in which a strong Coriolis interaction manifests. The model successfully describes the available intra- and interband $E 1, E 2$ and $M 1$ transition rates giving an insight into the mechanism which allows the $K$-suppressed transitions in axially symmetric nuclei.
\end{abstract}

\section{Introduction}

The role of the shape deformations in the manifestation of variety of dynamical effects in the structure of nuclear energy spectra and electromagnetic transitions is one of the continuously challenging problems in the theoretical and experimental nuclear structure study [1]. Among the most intriguing activities in this aspect are the efforts to understand the structure and dynamic properties of nuclei developed in the presence of reflection-asymmetric quadrupoleoctupole deformations [2].

The problem becomes even more challenging when referred to the interplay between collective and intrinsic degrees of freedom especially important in odd mass nuclei. This has been the subject of a model development involving the consideration of quadrupole-octupole vibration-rotation motion in the even-even core [3-6], the motion of the odd nucleon within a reflection-asymmetric axially-deformed potential [7] with pairing correlations of Bardeen-Cooper-Schrieffer (BCS) type implemented as in Ref. [8], and the Coriolis interaction between the core and the nucleon taken into account through the perturbation theory in a fully microscopic way [9]. It has been shown that within this formalism the spectra of oddmass nuclei with quadrupole-octupole degrees of freedom can be described in terms of yrast and non-yrast quasiparity-doublets (QPDs) - quadrupole-octupole vibrationrotation modes - built on one-quasiparticle excitations (bandheads) mixed though the Coriolis coupling [9]. It was demonstrated for the QPD energy levels of ${ }^{223} \mathrm{Ra}$ and ${ }^{221} \mathrm{Fr}$ that the model is capable to take into account both the Coriolis decoupling and $K$-mixing effects. However, the electromagnetic transition rates were not considered at this stage.

*e-mail: nminkov@inrne.bas.bg
Recently the model was applied to the low-lying spectrum of ${ }^{229} \mathrm{Th}$ in relation to the $7.8 \mathrm{eV}$ isomeric state supposing the presence of a soft axial quadrupole-octupole deformation [10]. The Coriolis $K$-mixing effect was taken into account within this formalism for the first time in order to predict the reduced $B(E 2)$ and $B(M 1)$ probabilities for a transition from the $K^{\pi}=3 / 2^{+}$isomeric state to the $K^{\pi}=5 / 2^{+}$ground state (g.s.) and to describe a number of observed intra- and interband transition rates in the ${ }^{229} \mathrm{Th}$ QPD spectrum. The result was obtained at relatively week Coriolis mixing strength and indicated the possibility for describing $K$-suppressed electromagnetic transitions in nuclei with a presence of axial symmetry.

In the present work we examine the aforementioned $K$-mixing formalism in the case of a much stronger Coriolis interaction observed in the QPD spectrum of ${ }^{221} \mathrm{Fr}$. Thus, we extend the study of Ref. [9] by including a number of $B(E 2), B(E 1)$ and $B(M 1)$ intra and interband transition rates into the model description of this nucleus. At the same time, we reformulate the model scheme by taking explicitly the single-particle (s.p.) energies of the bandheads obtained through the deformed shell model (DSM) [7] with BCS pairing interaction. It will be seen below that the model is not only capable to describe strong Coriolis mixing effects but also gives an insight into the fine interplay between collective and single-nucleon degrees of freedom which governs dynamical properties of nuclei with the presence of complex shape-deformation modes.

In the next Sec. 2, we present the main ingredients of the model applied. In Sec. 3, details of the application and model results for the QPD levels and transition rates in ${ }^{221} \mathrm{Fr}$ are given in comparison with experimental data. Concluding remarks are given in Sec. 4. 


\section{Model of quadrupole-octupole core plus particle}

The Hamiltonian of quadrupole-octupole (QO) vibrations and rotations coupled to the s.p. motion with Coriolis interaction and pairing correlations can be written in the form

$$
H=H_{\text {s.p. }}+H_{\text {pair }}+H_{\mathrm{QO}}+H_{\text {Coriol }} \text {. }
$$

Here, $H_{\text {s.p. }}$ is the s.p. Hamiltonian of the DSM with the Woods-Saxon (WS) potential for axial quadrupole, octupole and higher multipolarity deformations [7] providing the s.p. energies $E_{\text {s.p. }}^{K}$ with given value of the projection $K$ of the total and the s.p. angular momentum operators $\hat{I}$ and $\hat{j}$, respectively on the intrinsic symmetry axis. $H_{\text {pair }}$ is the standard BCS pairing Hamiltonian [11] which together with $H_{\text {s.p. }}$ determines the quasiparticle (q.p.) spectrum $\epsilon_{\text {q.p. }}^{K}$ as shown in Ref. [8]. $H_{\mathrm{QO}}$ represents oscillations of the even-even core with respect to the quadrupole $\left(\beta_{2}\right)$ and octupole $\left(\beta_{3}\right)$ axial deformation variables mixed through a centrifugal (rotation-vibration) interaction $[3,5]$. Its spectrum is obtained in an analytic form by assuming equal frequencies for the quadrupole and octupole oscillations known as coherent QO mode (CQOM). $H_{\text {Coriol }}$ involves the Coriolis interaction between the even-even core and the unpaired nucleon (see Eq. (3) in Ref. [5]). It is treated as a perturbation with respect to the remaining part of Hamiltonian (1) and then incorporated into the QO potential of $H_{\mathrm{QO}}$ defined for given angular momentum $I$, parity $\pi$ and the s.p. bandhead projection $K_{b}$ which leads to a joint term [9]

$$
\begin{aligned}
H_{\mathrm{QO}}^{I K_{b}}= & -\frac{\hbar^{2}}{2 B_{2}} \frac{\partial^{2}}{\partial \beta_{2}^{2}}-\frac{\hbar^{2}}{2 B_{3}} \frac{\partial^{2}}{\partial \beta_{3}^{2}}+\frac{1}{2} C_{2} \beta_{2}{ }^{2}+\frac{1}{2} C_{3} \beta_{3}{ }^{2} \\
& +\frac{\tilde{X}\left(I^{\pi}, K_{b}\right)}{d_{2} \beta_{2}^{2}+d_{3} \beta_{3}^{2}} .
\end{aligned}
$$

Here, $B_{2}\left(B_{3}\right), C_{2}\left(C_{3}\right)$, and $d_{2}\left(d_{3}\right)$ are quadrupole (octupole) mass, stiffness and inertia parameters, respectively. The function $\tilde{X}\left(I^{\pi}, K_{b}\right)$ determines the centrifugal term in which the Coriolis mixing is taken into account and has the form:

$$
\begin{aligned}
\tilde{X}\left(I^{\pi}, K_{b}\right) & =\frac{1}{2}\left[d_{0}+I(I+1)-K_{b}^{2}+(-1)^{I+\frac{1}{2}}\left(I+\frac{1}{2}\right)\right. \\
& \left.\times a^{\left(\pi, \pi^{b}\right)} \delta_{K_{b}, \frac{1}{2}}-A \sum_{\substack{v \neq b \\
\left(K_{v}=\frac{1}{2}, K_{b} \pm 1\right)}} \frac{\left[\tilde{a}_{K_{v} K_{b}}^{\left(\pi, \pi^{b}\right)}(I)\right]^{2}}{\epsilon_{\mathrm{q} . \mathrm{p} .}^{K_{v}}-\epsilon_{\mathrm{q} . \mathrm{p} .}^{K_{b}}}\right],
\end{aligned}
$$

where $d_{0}$ determines the QO potential origin, the quantity $a^{\left(\pi, \pi^{b}\right)}=\pi \cdot \pi^{b} a_{1 / 2,-1 / 2}^{\left(\pi^{b}\right)}$ represents the decoupling factor for the case $K_{b}=1 / 2$ and $\tilde{a}_{K_{v} K_{b}}^{\left(\pi, \pi^{b}\right)}$ represent the Coriolis mixing factors:

$$
\tilde{a}_{K_{v} K_{b}}^{\left(\pi, \pi^{b}\right)}(I)=\left\{\begin{array}{cc}
\sqrt{\left(I-K_{b}\right)\left(I+K_{b}+1\right)} a_{K_{v} K_{b}}^{\left(\pi^{b}\right)}, & K_{v}=K_{b}+1 \\
\sqrt{\left(I+K_{b}\right)\left(I-K_{b}+1\right)} a_{K_{b} K_{v}}^{\left(\pi_{v}^{b},\right.} & K_{v}=K_{b}-1 \\
\pi \cdot \pi^{b}(-1)^{\left(I+\frac{1}{2}\right)}\left(I+\frac{1}{2}\right) a_{\frac{1}{2},-\frac{1}{2}}^{\left(\pi^{b}\right)}, & K_{v}=K_{b}=\frac{1}{2}
\end{array}\right.
$$

with

$$
\begin{aligned}
a_{K_{v} K_{b}}^{\left(\pi^{b}\right)} & =\frac{P_{K_{v} K_{b}}^{b}}{N_{K_{v}}^{\left(\pi^{b}\right)} N_{K_{b}}^{\left(\pi^{b}\right)}}\left\langle\mathcal{F}_{K_{v}}^{\left(\pi^{b}\right)}\left|\hat{j}_{+}\right| \mathcal{F}_{K_{b}}^{\left(\pi^{b}\right)}\right\rangle \\
& =\frac{P_{K_{b} K_{v}}^{b}}{N_{K_{b}}^{\left(\pi^{b}\right)} N_{K_{v}}^{\left(\pi^{b}\right)}}\left\langle\mathcal{F}_{K_{b}}^{\left(\pi^{b}\right)}\left|\hat{j}_{-}\right| \mathcal{F}_{K_{v}}^{\left(\pi^{b}\right)}\right\rangle .
\end{aligned}
$$

The latter involve matrix elements of the s.p. operators $\hat{j}_{ \pm}=\hat{j}_{x} \pm i \hat{j}_{y}$ between the parity-projected components of the s.p. wave functions $\mathcal{F}_{K_{b}}^{\left(\pi^{b}\right)}$ of the bandhead state and the admixing state $\mathcal{F}_{K_{y}}^{\left(\pi^{b}\right)}$ determined by DSM [7]. Each s.p. wave function, which has a mixed parity due to the reflection asymmetry, is projected on the experimentally assigned good parity $\pi^{b}$ of the bandhead s.p. state. The quantity $N_{K}^{\left(\pi^{b}\right)}=\left[\left\langle\mathcal{F}_{K}^{\left(\pi^{b}\right)} \mid \mathcal{F}_{K}^{\left(\pi^{b}\right)}\right\rangle\right]^{1 / 2}$ is a parity-projected normalization factor, whereas $P_{K_{\gamma^{\prime}} K_{v}}^{b}=U_{K_{\gamma^{\prime}}}^{b} U_{K_{v}}^{b}+V_{K_{\gamma^{\prime}}}^{b} V_{K^{\prime}}^{b}$ involves the BCS occupation factors. The index $b$ corresponds to the blocked s.p. orbital on which the collective spectrum is built. Since the BCS procedure is performed separately for each (blocked) bandhead orbital the overlap integrals and the matrix elements between states built on different bandhead orbitals involve the average of both separate occupation factors $P_{K_{v^{\prime}} K_{v}}^{b b^{\prime}}=\frac{1}{2}\left(P_{K_{v^{\prime}} K_{v}}^{b}+P_{K_{v} K_{v^{\prime}}}^{b^{\prime}}\right)$. The sum in Eq. (3) runs over q.p. states with energies $\epsilon_{\mathrm{q} . \mathrm{p}}^{K_{v}}$ above the Fermi level and $A$ is the Coriolis mixing strength defined in [9]. In our numerical calculations we consider ten mixing orbitals.

In this consideration the spectrum which corresponds to Hamiltonian (1) represents QO vibrations and rotations built on a q.p. state with $K=K_{b}$ and parity $\pi^{b}$. The corresponding energy expression has the form [9]

$$
E_{n k}^{\mathrm{tot}}\left(I^{\pi}, K_{b}\right)=\epsilon_{\mathrm{q} . \mathrm{p} .}^{K_{b}}+\hbar \omega\left[2 n+1+\sqrt{k^{2}+b \tilde{X}\left(I^{\pi}, K_{b}\right)}\right],
$$

where $b=2 B /\left(\hbar^{2} d\right)$ denotes the reduced inertia parameter and $n=0,1,2, \ldots$ and $k=1,2, \ldots$ stand for the radial and angular QO oscillation quantum numbers, respectively, with $k$ odd (even) for the even (odd) parity states of the core [3]. The levels of the total QO core plus particle system, determined by a particular $n$ and $k^{(+)}\left(k^{(-)}\right)$ for the states with given $I^{\pi=+}\left(I^{\pi=-}\right)$ form a split doublet with respect to the parity, QPD [6]. Furthermore, $\omega=\sqrt{C_{2} / B_{2}}=\sqrt{C_{3} / B_{3}} \equiv \sqrt{C / B}$ stands for the frequency of the coherent QO oscillations and $d=\left(d_{2}+d_{3}\right) / 2[4,6]$.

The Coriolis perturbed wave function corresponding to Hamiltonian (1) with the spectrum, Eq. (6), is obtained in the first order of perturbation theory and has the form

$$
\begin{aligned}
\tilde{\Psi}_{n k I M K_{b}}^{\left(\pi, \pi^{b}\right)} & =\frac{1}{\tilde{N}_{I \pi K_{b}}}\left[\Psi_{n k I M K_{b}}^{\left(\pi, \pi^{b}\right)}\right. \\
& \left.+A \sum_{\substack{v \neq b \\
\left(K_{v}=K_{b} \pm 1, \frac{1}{2}\right)}} C_{K_{v} K_{b}}^{I \pi} \Psi_{n k I M K_{v}}^{\left(\pi, \pi^{b}\right)}\right],
\end{aligned}
$$

where the expansion coefficients are given by $C_{K_{v} K_{b}}^{I \pi}=\tilde{a}_{K_{v} K_{b}}^{\left(\pi, \pi^{b}\right)}(I) /\left(\epsilon_{\mathrm{q} . \mathrm{p} .}^{K_{v}}-\epsilon_{\mathrm{q} . \mathrm{p} .}^{K_{b}}\right)$ and $\tilde{N}_{I \pi K_{b}}^{2}=\left\langle\tilde{\Psi}_{n k I M K_{b}}^{\left(\pi, \pi^{b}\right)} \mid \tilde{\Psi}_{n k I M K_{b}}^{\left(\pi, \pi^{b}\right)}\right\rangle$ 
is the normalization constant of the form

$$
\begin{aligned}
& \tilde{N}_{I \pi K_{b}}^{2}=1+2 A \sum_{\substack{v \neq b \\
\left(K_{\nu}=K_{b}=\frac{1}{2}\right)}} C_{K_{v} K_{b}}^{I \pi} \delta_{K_{v}, K_{b}} \\
& \times \frac{P_{K_{v} K_{b}}^{b}}{N_{K_{v}}^{\left(\pi^{b}\right)} N_{K_{b}}^{\left(\pi^{b}\right)}}\left\langle\mathcal{F}_{K_{v}}^{\left(\pi^{b}\right)} \mid \mathcal{F}_{K_{b}}^{\left(\pi^{b}\right)}\right\rangle \\
& +A^{2} \sum_{\substack{v_{1} \neq b \\
\left(K_{v_{1}}=K_{b} \pm 1, \frac{1}{2}\right)}} \sum_{\substack{\nu_{2} \neq b \\
\left(K_{\nu_{2}}=K_{b} \pm 1, \frac{1}{2}\right)}} C_{K_{\nu_{1}} K_{b}}^{I \pi} C_{K_{v_{2}} K_{b}}^{I \pi} \delta_{K_{\nu_{1}}, K_{\nu_{2}}} \\
& \times \frac{P_{K_{\nu_{1}} K_{\nu_{2}}}^{b}}{N_{K_{\nu_{1}}}^{\left(\pi^{b}\right)} N_{K_{v_{2}}}^{\left(\pi^{b}\right)}}\left\langle\mathcal{F}_{K_{\nu_{1}}}^{\left(\pi^{b}\right)} \mid \mathcal{F}_{K_{v_{2}}}^{\left(\pi^{b}\right)}\right\rangle
\end{aligned}
$$

The unperturbed QO core plus particle wave function in Eq. (7) has the form [9]

$$
\begin{array}{r}
\Psi_{n k I M K}^{\left(\pi, \pi^{b}\right)}(\eta, \phi, \theta)=\frac{1}{N_{K}^{\left(\pi^{b}\right)}} \sqrt{\frac{2 I+1}{16 \pi^{2}}} \Phi_{n k I}^{\left(\pi, \pi^{b}\right)}(\eta, \phi) \\
\times\left[D_{M K}^{I}(\theta) \mathcal{F}_{K}^{\left(\pi^{b}\right)}+\pi \cdot \pi^{b}(-1)^{I+K} D_{M-K}^{I}(\theta) \mathcal{F}_{-K}^{\left(\pi^{b}\right)}\right],
\end{array}
$$

where $D_{M K}^{I}(\theta)$ are the rotation (Wigner) functions and $\Phi_{n k I}^{\left(\pi, \pi^{b}\right)}(\eta, \phi)$ are the QO vibration functions obtained after solving the Schrödinger equation for the Hamiltonian (2) in radial $(\eta)$ and angular $(\phi)$ coordinates (see Refs. $[4,6]$ for details).

Having the Coriolis perturbed wave function (7) and using the theory for electric transition probabilities in CQOM developed in [4] we were able to calculate the reduced probabilities $B(E 1), B(E 2)$ and $B(E 3)$ for transitions between initial $(i)$ and final $(f)$ states with energies given by Eq. (6). Also, by using the standard form of the $M 1$ operator given in Ref. [11] (see, e.g., Eq. (3.61) therein) we have obtained an expression for the respective $B(M 1)$ transition probability. It appears that the expressions for both types $(T)$, electric $(T=E)$ and magnetic $(T=M)$ transition with multipolarity $\lambda$ can be derived in a common form

$$
\begin{aligned}
& B\left(T \lambda ; \pi^{b_{i}} I_{i} \pi_{i} K_{i} \rightarrow \pi^{b_{f}} I_{f} \pi_{f} K_{f}\right) \\
& =R^{T \lambda} \delta_{\pi^{b_{f}}, \pi^{b_{i}}}\left[\left(1+\pi_{f} \cdot \pi_{i}(-1)^{\lambda \delta_{T, E}}\right) / 2\right] \\
& \times \frac{1}{\tilde{N}_{I_{f} \pi_{f} K_{f}}^{2} \tilde{N}_{I_{i} \pi_{i} K_{i}}^{2}}\left[\delta_{K_{f}, K_{i}} C_{I_{i} K_{i} \lambda 0}^{I_{f} K_{f}} \frac{\left.P_{K_{f} K_{i}}^{b_{f} b_{i}} M_{K_{f} K_{i}}^{\left(\pi^{b} f\right.} \pi^{b_{i}}\right)}{N_{K_{f}}^{\left(\pi^{b} f\right)} N_{K_{i}}^{\left(\pi^{b_{i}}\right)}}\right. \\
& +A C_{I_{i} K_{f} \lambda 0}^{I_{f} K_{f}} \sum_{\substack{v \neq i \\
\left(K_{v}=K_{i} \pm 1, \frac{1}{2}\right)}} \delta_{K_{f}, K_{\nu}} C_{K_{v} K_{i} \pi_{i}}^{I_{i}} \frac{P_{K_{f} K_{v}}^{b_{f}} M_{K_{f} K_{v}}^{\left(\pi^{b} f\right.} \pi^{\left.b_{i}\right)}}{N_{K_{f}}^{\left(\pi^{b}\right)} N_{K_{v}}^{\left(\pi^{b_{i}}\right)}} \\
& +A C_{I_{i} K_{i} \lambda 0}^{I_{f} K_{i}} \sum_{\substack{v \neq f \\
\left(K_{v}=K_{f} \pm 1, \frac{1}{2}\right)}} \delta_{K_{v}, K_{i}} C_{K_{v} K_{f}}^{I_{f} \pi_{f}} \frac{P_{K_{v} K_{i}}^{b_{i}} M_{K_{v} K_{i}}^{\left(\pi^{b_{f}}, \pi^{b_{i}}\right)}}{N_{K_{v}}^{\left(\pi^{b}\right)} N_{K_{i}}^{\left(\pi^{b_{i}}\right)}} \\
& +A^{2} \sum_{\substack{v^{\prime \prime} \neq f \\
\left(K_{v^{\prime \prime}}=K_{f} \pm 1, \frac{1}{2}\right)}} \sum_{\substack{v^{\prime} \neq i \\
\left(K_{v^{\prime}}=K_{i} \pm 1, \frac{1}{2}\right)}} \delta_{K_{v^{\prime \prime}}, K_{\nu^{\prime}}} C_{I_{i} K_{v^{\prime}}}^{I_{f} K_{\nu^{\prime \prime}}} \lambda K_{v^{\prime \prime}}-K_{v^{\prime}} \\
& \left.\times C_{K_{v^{\prime \prime}} K_{f}}^{I_{f} \pi_{f}} C_{K_{\nu^{\prime}} I_{i} \pi_{i}}^{I_{i}} \frac{\left.\left.P_{K_{\nu^{\prime \prime}} K_{v^{\prime}}}^{b_{f} b_{i}} M_{K_{\nu^{\prime \prime}} K_{v^{\prime}}}^{\left(\pi^{b} f\right.}\right]^{b_{i}}\right)}{N_{K_{v^{\prime \prime}}}^{\left(\pi^{b} f\right)} N_{K_{v^{\prime}}}^{\left(\pi^{b_{i}}\right)}}\right]^{2}
\end{aligned}
$$

where

$$
R^{T \lambda=E \lambda}=\frac{2 \lambda+1}{4 \pi\left(4-3 \delta_{\lambda, 1}\right)} R_{\lambda}^{2}\left(\pi^{b_{i}} n_{i} k_{i} I_{i} \rightarrow \pi^{b_{f}} n_{f} k_{f} I_{f}\right)
$$

involves integrals on the radial and angular variables in CQOM (see Eqs. (35)-(41) and Appendices B and C in Ref. [4]) and

$$
R^{T 1=M 1}=\frac{3}{4 \pi} \mu_{N}^{2}
$$

involves the nuclear magneton. Also here

$$
M_{K_{f} K_{i}}^{\left(\pi^{b_{f}}, \pi^{b_{i}}\right)}= \begin{cases}\left\langle\mathcal{F}_{K_{f}}^{\left(\pi^{b_{f}}\right)} \mid \mathcal{F}_{K_{i}}^{\left(\pi^{b_{i}}\right)}\right\rangle, & T=E \\ {\left[\left(g_{l}-g_{R}\right) K_{i} \delta_{K_{f}, K_{i}}\left\langle\mathcal{F}_{K_{f}}^{\left(\pi^{b_{f}}\right)} \mid \mathcal{F}_{K_{i}}^{\left(\pi^{b_{i}}\right)}\right\rangle\right.} & \\ \left.+\left(g_{s}-g_{l}\right)\left\langle\mathcal{F}_{K_{f}}^{\left(\pi^{b_{f}}\right)}\left|\hat{s}_{z}\right| \mathcal{F}_{K_{i}}^{\left(\pi^{b_{i}}\right)}\right\rangle\right], & T=M\end{cases}
$$

where $g_{R}=Z / A_{\text {mass }}$ is the rotation gyromagnetic factor, $\hat{s}_{z}$ is the $z$-component of the spin operator, $g_{l}=0(1)$ for neutrons (protons) is the orbital gyromagnetic factor, whereas the spin gyromagnetic factor is $g_{s}=0.6 g_{s}^{\text {free }}$ with $g_{s}^{\text {free }}=$ -3.826 (5.586) for neutrons (protons). The symbol $C_{I_{1} K_{1} \lambda \mu}^{I_{2} K_{2}}$ denotes the Clebsch-Gordan coefficient.

We note that the reduced transition probability expression, Eq. (10), contains first-order and second-order $K$ mixing effects. First-order mixing terms practically contribute with nonzero values only in the cases $K_{i / f}=K_{v}=1 / 2$, i.e., when a $K_{i / f}=1 / 2$ bandhead state is mixed with another $K_{v}=1 / 2$ state present in the considered range of admixing orbitals above the Fermi level. A second-order mixing effect connects states with $\Delta K=1,2$ and allows different combinations of $\left|K_{i}-K_{f}\right| \leq 2$ which provide respective nonzero contribution of the Coriolis mixing to the transition probability. In this way the present formalism provides nonzero transition probabilities between states with different $K$-values despite the axial symmetry assumed in both CQOM and DSM parts of Hamiltonian (1).

\section{QPD spectrum and transition rates with strong Coriolis interaction in ${ }^{221} \mathrm{Fr}$}

Here we demonstrate the application of the above core plus particle scheme on the QPD spectrum in ${ }^{221} \mathrm{Fr}$. The result is given in Fig. 1.

At the first step we determine the quadrupole and octupole deformation values $\beta_{2}$ and $\beta_{3}$ entering DSM so as to provide the necessary s.p. orbitals for the ground and excited bandhead states with $K=1 / 2$ and $3 / 2$, respectively, with negative sign of the respective average parities $\left\langle\pi^{b}\right\rangle<0$ and large enough decoupling factor value for the yrast QPD as well. In practice we have tested various regions of $\left(\beta_{2}, \beta_{3}\right)$ deformations similarly to the study illustrated in Fig. 8.7 in Ref. [12] (see page 312 therein). In addition, we entered and slightly varied a nonzero value of the hexadecapole deformation $\beta_{4}$. Thus, we determined $\beta_{2}=0.096, \beta_{3}=0.075$ and $\beta_{4}=0.001$ for which the s.p. orbital of the g.s. is $1 / 2$ [510] with average parity $\left\langle\pi^{b}\right\rangle=-0.223$, while that for the excited QPD is $3 / 2$ [521] with $\left\langle\pi^{b}\right\rangle=-0.254$. At these conditions we obtain the yrast band decoupling factor through Eqs. (4) and (5) with the 


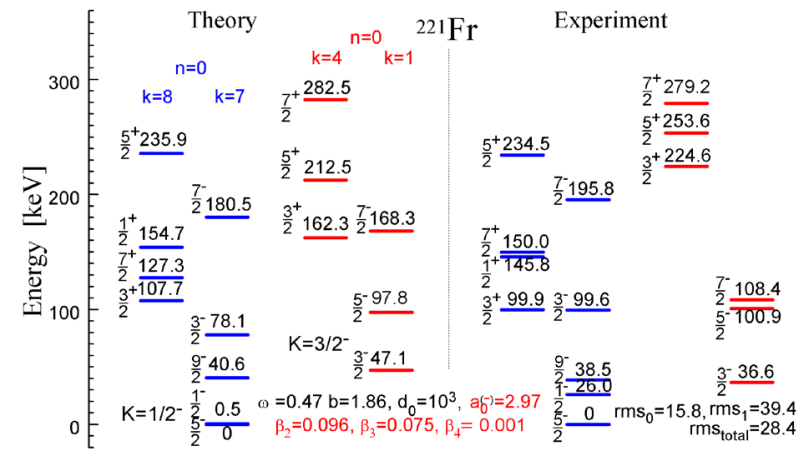

Figure 1. (Color online) Theoretical and experimental QPD spectrum in ${ }^{221} \mathrm{Fr}$. The units of the CQOM parameters are: $\mathrm{MeV} / \hbar$ for $\omega, \hbar^{-2}$ for $b$ and $\hbar^{2}$ for $d_{0}$. The experimental data are taken from Ref. [13].

value $a_{0}^{(-)}=\pi \cdot \pi^{b} a_{1 / 2,-1 / 2}^{\left(\pi^{b}\right)}=2.97$. The BCS procedure is applied with the pairing constants determined as explained below Eq. (4) of Ref. [8] and provides the q.p. energy $\epsilon_{\mathrm{q} . \mathrm{p} .}^{K_{b}}=376 \mathrm{keV}$ for the $3 / 2[521]$ orbital.

On the above basis we consider two QPDs, namely the yrast (yr) one built on the $K_{b^{\mathrm{yr}}}^{\pi}=1 / 2^{-}$g.s. corresponding to the 1/2[510] s.p. orbital and the non-yrast, excited (ex), QPD built on the $K_{b^{\text {ex }}}^{\pi}=3 / 2^{-}$q.p. state corresponding to the 3/2[521] s.p. orbital.

To determine the relevant quadrupole-octupole vibration-rotation modes at the next step we have examined various model QPD structures corresponding to different sets of oscillator quantum numbers $n$ and $\left(k^{(+)}, k^{(-)}\right)$. Similarly, to the case of ${ }^{229} \mathrm{Th}$ [10] we found that both quasidoublets should have the same value $n=0$ of the radial QO oscillator quantum number with different pairs of angular $\left(k^{(+)}, k^{(-)}\right)$values for each QPD. The latter were determined so as to provide the best model description of the energy levels together with the attendant transition rates after adjusting the CQOM parameters and the Coriolis mixing constant for each different set of $\left(k^{(+)}, k^{(-)}\right)$ values. The values of the QO oscillator quantum numbers and CQOM parameters obtained in the fit which provide the final result for the model energies are given in Fig. 1 with the adjusted Coriolis mixing constant taking the value $A=110.3 \mathrm{keV}$. It is seen that the angular quantum numbers $\left(k^{(+)}, k^{(-)}\right)=(8,7)$ obtained for the QO mode superposed on the $1 / 2$ [510] g.s. orbital are larger than the ones $(4,1)$ obtained for the QO mode built on the $3 / 2$ [521] orbital. This suggests a stronger influence of the QO mode on the s.p. motion in the $1 / 2^{-}$g.s. with a larger energy contribution [see Eq. (6)] compared to that in the $3 / 2^{-}$excitation. As a result, after deduction of the g.s. energy the $3 / 2^{-}$bandhead appears much lower than the original DSM q.p. energy of $376 \mathrm{keV}$. This allows the model to reproduce relatively well the experimental $3 / 2^{-}$bandhead energy of $\sim 37 \mathrm{keV}$.

It should be remarked that while the $\beta_{2}$ and $\beta_{3}$ deformation parameters determining the s.p. motion in DSM are fixed (the values given above) the collective coherent QO motion does not correspond to fixed deformations and only imposes certain limits in which the "dynamical" deformation modes evolve. The latter can be deduced from the two-dimensional QO potential shapes illustrated in Figs. 3 and 5 of Ref. [3] and their ellipsoidal bottoms (minima) shown in Fig. 4 there, as well as, in Fig. 9 of Ref. [4]. It is seen that depending on the particular coherent QO excitation (see Fig. 9 in Ref. [4]) the maxima of the wave function density corresponding to dynamical $\beta_{2}$ and $\beta_{3}$ deformations appear between zero and some maximal deformation values determined by the semiaxes (s.a.) of the potential-bottom ellipsoid. This means that the present approach suggests much softer QO deformation modes of the system in the collective potential than for the s.p. motion in the deformed WS potential. For the collective parameters determined in the present calculation the semiaxes of the QO potential are obtained as $\beta_{2}^{\text {s.a. }}=0.15$ and $\beta_{3}^{\text {s.a. }}=0.23$ for the g.s. angular momentum (see Eq. (46) in [4]). This means that the deformation values appearing on the collective potential bottom surround the $\left(\beta_{2}, \beta_{3}\right)$ deformations entering DSM. In addition, these values suggest that the nucleus ${ }^{221}$ Fr may be softer against the octupole deformation than against the quadrupole deformation.

In Fig. 1, the theoretically obtained energy levels are compared with the experimental ones. It is seen that the model-determined QPD spectrum of ${ }^{221}$ Fr reasonably reproduces the structure of the experimental spectrum. The root-mean-square (rms) deviation of the predicted energy levels from the experimental ones is $\mathrm{rms}_{0}=15.8 \mathrm{keV}$ for the yrast-based band and $\mathrm{rms}_{1}=39.4 \mathrm{keV}$ for the non-yrast band, with the total deviation being $\mathrm{rms}_{\text {total }}=28.4 \mathrm{keV}$. The theoretically determined Coriolis decoupling factor together with the $K$-mixing effect allow us to describe the reordering of the angular momentum sequences in the yrast $K_{b^{\mathrm{yr}}}^{\pi}=1 / 2^{-}$QPD. We see that in the negativeparity sequence the experimental reordering is reproduced while in its positive-parity counterpart only the place of the $7 / 2^{+}$state, which is pushed a bit lower, does not correspond to the experimental one. It should be noted that compared to ${ }^{229}$ Th here the Coriolis decoupling/mixing effect is much stronger and even exceeds for some levels the usual assumption of small perturbation (see in Ref. [9] for detailed analysis in this aspect). Therefore, the present result for ${ }^{221}$ Fr shows that the applied CQOM+DSM approach is capable to take into account the influence of the strong Coriolis interaction on the QPD energy levels.

Regarding the electromagnetic transition rates the approach can be assessed by the result shown in Table 1 . There a number of intra- and inter- QPD band $B(E 1)$, $B(E 2)$ and $B(M 1)$ transition rates are described by fitting three additional parameters (given in the Table 1) together with the energy parameters to the experimental data. It is seen that most of the intra- QPD band transition rates are reproduced quite well. Some of the inter- QPD band rates such as $B\left(E 1 ; 3 / 2_{\mathrm{ex}}^{+} \rightarrow 1 / 2_{\mathrm{yr}}^{-}\right)=1.3 \times 10^{-4}$ W.u. (theory), $1.2 \times 10^{-4}$ W.u. (experiment) are described very well, others such as $B\left(M 1 ; 3 / 2_{\mathrm{yr}}^{-} \rightarrow 3 / 2_{\mathrm{ex}}^{-}\right), B\left(M 1 ; 7 / 2_{\mathrm{ex}}^{-} \rightarrow 5 / 2_{\mathrm{yrs}}^{-}\right)$are reasonably described whereas for some others the difference between the theory and experiment goes up to an order. Despite of the latter the result shows the principal 
Table 1. Theoretical and experimental transition rates for ${ }^{221} \mathrm{Fr}$. The CQOM parameter values adjusted to experimental data on transitions are $c=10^{3}, p=0.83$ and $E 1$ effective charge $e_{\mathrm{eff}}^{1}=0.8$ (all dimensionless). The experimental data are taken from [14].

\begin{tabular}{cccc}
\hline Type/Mult. & Transition & Theory (W.u.) & Experiment (W.u.) \\
\hline$E 2$ & $3 / 2_{\mathrm{yr}}^{-} \rightarrow 5 / 2_{\mathrm{yr}}^{-}$ & 25.9 & $23( \pm 16)$ \\
$E 2$ & $7 / 2_{\mathrm{yr}}^{-} \rightarrow 5 / 2_{\mathrm{yr}}^{-}$ & 14.9 & $16( \pm 8)$ \\
$E 1$ & $7 / 2_{\mathrm{yr}}^{+} \rightarrow 5 / 2_{\mathrm{yr}}^{-}$ & 0.00014 & $0.00038( \pm 0.00010)$ \\
$E 1$ & $7 / 2_{\mathrm{yr}}^{+} \rightarrow 9 / 2_{\mathrm{yr}}^{-}$ & 0.00018 & $0.00041( \pm 0.00011)$ \\
$E 2$ & $7 / 2_{\mathrm{ex}}^{-} \rightarrow 3 / 2_{\mathrm{ex}}^{-}$ & 61.8 & $\approx 55$ \\
$E 1$ & $3 / 2_{\mathrm{ex}}^{+} \rightarrow 5 / 2_{\mathrm{ex}}^{-}$ & 0.00083 & $0.00021( \pm 0.00007)$ \\
$E 1$ & $3 / 2_{\mathrm{ex}}^{+} \rightarrow 3 / 2_{\mathrm{ex}}^{-}$ & 0.00080 & $0.00038( \pm 0.00011)$ \\
$E 1$ & $5 / 2_{\mathrm{ex}}^{+} \rightarrow 3 / 2_{\mathrm{ex}}^{-}$ & 0.00055 & $0.00017( \pm 0.00012)$ \\
$E 2$ & $7 / 2_{\mathrm{ex}}^{-} \rightarrow 5 / 2_{\mathrm{yr}}^{-}$ & 0.14 & $\approx 26$ \\
$E 2$ & $3 / 2_{\mathrm{ex}}^{+} \rightarrow 3 / 2_{\mathrm{yrs}}^{+}$ & 1.12 & $\approx 68$ \\
$E 1$ & $3 / 2_{\mathrm{ex}}^{+} \rightarrow 1 / 2_{\mathrm{yr}}^{-}$ & 0.000013 & $0.000012( \pm 0.000004)$ \\
$E 1$ & $5 / 2_{\mathrm{ex}}^{+} \rightarrow 5 / 2_{\mathrm{rs}}^{-}$ & 0.0000027 & $0.000045( \pm 0.000022)$ \\
$M 1$ & $3 / 2_{\mathrm{yr}}^{-} \rightarrow 5 / 2_{\mathrm{yr}}^{-}$ & 0.0495 & $0.022( \pm 0.009)$ \\
$M 1$ & $3 / 2_{\mathrm{yr}}^{-} \rightarrow 3 / 2_{\mathrm{ex}}^{-}$ & 0.022 & $0.056( \pm 0.022)$ \\
$M 1$ & $7 / 2_{\mathrm{ex}}^{-} \rightarrow 5 / 2_{\mathrm{yr}}^{-}$ & 0.0017 & $\approx 0.0033$ \\
$M 1$ & $7 / 2_{\mathrm{yr}}^{-} \rightarrow 9 / 2_{\mathrm{yr}}^{\mathrm{y}}$ & 0.099 & $0.023( \pm 0.007)$ \\
$M 1$ & $3 / 2_{\mathrm{ex}}^{+} \rightarrow 3 / 2_{\mathrm{yr}}^{+}$ & 0.022 & $\approx 0.005$ \\
$M 1$ & $3 / 2_{\mathrm{ex}}^{+} \rightarrow 1 / 2_{\mathrm{yr}}^{+}$ & 0.0044 & $0.015( \pm 0.005)$
\end{tabular}

capability of the model to reproduce non-zero experimental transition rates between states with different $K$-values which would be obtained with zero values if the Coriolis mixing was not taken into account.

We should remark that the above described effects of Coriolis decoupling and $K$-mixing interaction on the energy spectrum and electromagnetic transition rates in ${ }^{221} \mathrm{Fr}$ are essentially determined by the collective shapedeformation modes in this nucleus. Both, the decoupling parameter in the yrast QPD band and the $K$-mixing coefficients determined through Eq. (4) depend on the particular values of the quadrupole, octupole and higher multipolarity deformation modes such as the hexadecapole one. Moreover, the octupole deformation entering DSM involves parity mixing effects which after the projection considerably influence the structure of the core plus particle wave function and subsequently the electromagnetic transition characteristics of the nucleus.

\section{Concluding remarks}

In conclusion, we have shown that by taking into account in a proper model scheme, such as the considered CQOM+DSM approach, the influence of the complex shape-deformation modes on the interaction between the collective and s.p. degrees of freedom in the nucleus one may be able to get an insight into fine effects in the manifestation of nuclear energy and electromagnetic transition properties. We have demonstrated that the Coriolis $K$-mixing mechanism in combination with the collective quadrupole-octupole shape dynamics may govern the manifestation of $K$-suppressed transitions in the spectra of nuclei with overall axial symmetry. The present result shows the need for a systematic study in the above aspect of the Coriolis mixing effects in more nuclei and nuclear regions. Also, the $K$-mixing mechanism, as implemented in the presented model formalism, may be of a special use in the study of interesting phenomena such as the $K$-isomer decay modes of atomic nuclei. This is the subject of further work.

This work has been supported by the Bulgarian National Science Fund under contract no. DFNI-E02/6.

\section{References}

[1] J.M. Eisenberg, W. Greiner, Nuclear Theory: Nuclear Models, 3rd ed., (North-Holland, Amsterdam, 1987), Vol. 1.

[2] P.A. Butler, W. Nazarewicz, Rev. Mod. Phys. 68, 349 (1996)

[3] N. Minkov, P. Yotov, S. Drenska, W. Scheid, D. Bonatsos, D. Lenis, D. Petrellis, Phys. Rev. C 73, 044315 (2006)

[4] N. Minkov, S. Drenska, M. Strecker, W. Scheid, H. Lenske, Phys. Rev. C 85, 034306 (2012)

[5] N. Minkov, S. Drenska, P. Yotov, S. Lalkovski, D. Bonatsos, W. Scheid, Phys. Rev. C 76, 034324 (2007)

[6] N. Minkov, S. Drenska, K. Drumev, M. Strecker, H. Lenske, W. Scheid, Phys. Rev. C 88, 064310 (2013)

[7] S. Cwiok, J. Dudek, W. Nazarewicz, J. Skalski, T. Werner, Comput. Phys. Commun. 46, 379 (1987)

[8] P.M. Walker, N. Minkov, Phys. Lett. B 694, 119 (2010)

[9] N. Minkov, Phys. Scr. T154, 014017 (2013)

[10] N. Minkov, A. Pálffy, Phys. Rev. Lett. 118, 212501 (2017) 
[11] P. Ring, P. Schuck, The Nuclear Many-Body Problem (Springer-Verlag, Berlin, 1980)

[12] N. Minkov, Models of Complex Deformed Atomic Nuclei, (D.Sc. Thesis, Institute of Nuclear Research and Nuclear Energy, Bulgarian Academy of Sci- ences, Sofia, 2018)

[13] National Nuclear Data Center, [http://www.nndc.bnl. gov/ensdf/]

[14] National Nuclear Data Center, [http://www.nndc.bnl. gov/nudat2/indx_adopted.jsp] 